\title{
Effect of type of TAG fatty acids on lutein and zeaxanthin bioavailability
}

\author{
Béatrice Gleize ${ }^{1,2,3}$, Franck Tourniaire ${ }^{1,2,3}$, Laurence Depezay ${ }^{4}$, Romain Bott ${ }^{1,2,3}$, Marion Nowicki ${ }^{1,2,3}$, \\ Lionel Albino ${ }^{4}$, Denis Lairon ${ }^{1,2,3}$, Emmanuelle Kesse-Guyot ${ }^{5}$, Pilar Galan ${ }^{5}$, Serge Hercberg ${ }^{5}$ and \\ Patrick Borel ${ }^{1,2,3 *}$ \\ ${ }^{1}$ INRA, UMR1260, Research Unit in Nutrition, Obesity and Risk of Thrombosis, Faculté de Médecine, 27 Boulevard \\ Jean-Moulin, F-13385 Marseille, Cedex 5, France \\ ${ }^{2}$ INSERM, UMR1062, Marseille F-13385, France \\ ${ }^{3}$ Aix-Marseille Université, Marseille F-13385, France \\ ${ }^{4}$ Bonduelle, Villeneuve d'Ascq F-59653, France \\ ${ }^{5}$ Nutritional Epidemiology Research Unit (UREN), INSERM U557, INRA U1125, CNAM, Université Paris 13, Sorbonne Paris \\ Cité, F-93017 Bobigny Cedex, France
}

(Submitted 23 May 2012 - Final revision received 17 September 2012 - Accepted 22 September 2012 - First published online 11 December 2012)

\begin{abstract}
The xanthophylls lutein and zeaxanthin probably play a role in visual function and may participate in the prevention of age-related eye diseases. Although a minimum amount of TAG is required for an optimal bioavailability of these carotenoids, the effect of the type of TAG fatty acids (FA) is less clear. The aim was to assess the effect of the type of TAG FA on bioavailability of these xanthophylls. A total of three complementary models were used: an in vitro digestion model to study bioaccessibility, Caco-2 cells to study uptake efficiency and orally administered rats to study in vivo bioavailability. Results showed that lutein and zeaxanthin bioaccessibility was greater (about $20-30 \%$, $P<0.05)$ with butter and palm oil than with olive and fish oils. Mixed micelle size, which was significantly lower (about $8 \%, P<0.05)$ with SFA than with unsaturated FA, was inversely related to lutein and zeaxanthin bioaccessibility. There was no significant effect of the type of TAG FA on xanthophyll uptake by Caco-2 cells, but some compounds present in natural oils significantly affected xanthophyll uptake. Oral administration of rats with spinach and butter over $3 \mathrm{~d}$ led to a higher fasting plasma lutein concentration than oral administration with olive or fish oils. In conclusion, dietary fats rich in SFA lead to a higher bioavailability of lutein and zeaxanthin, as compared with fats rich in MUFA and PUFA. This is due partly to the higher bioaccessibility of these xanthophylls in the smaller mixed micelles produced when SFA are incorporated into mixed micelles.
\end{abstract}

Key words: Xanthophyll: Bioavailability: SFA: Unsaturated fatty acids

Carotenoids are pigments responsible for the yellow, orange and red colours found in fruit, vegetables, algae and fungi. More than 650 carotenoids have been described and isolated from natural sources; however, only about fifty carotenoids can be detected in human blood and tissues ${ }^{(1)}$. Carotenoids are classified as hydrocarbon carotenoids or carotenes (e.g. $\beta$-carotene, $\alpha$-carotene and lycopene) and oxy-carotenoids or xanthophylls (e.g. lutein, zeaxanthin and $\beta$-cryptoxanthin). The consumption of foods rich in carotenoids has been associated with a decrease in the incidence of some human degenerative and chronic diseases ${ }^{(2,3)}$. Lutein and zeaxanthin are gaining increasing interest as they selectively accumulate in the human retina ${ }^{(4)}$, where they apparently protect photoreceptors against light-initiated oxidative damage ${ }^{(5,6)}$, and because they have been associated with a lower incidence of age-related macular degeneration ${ }^{(7,8)}$.

In order to achieve their potential beneficial health effects, xanthophylls must be efficiently absorbed and carried to their target tissues. Absorption of xanthophylls, like other lipophilic compounds, is ensured through three key limiting steps: (1) release from the food matrix (usually a vegetable matrix) and transfer into the mixed micelles during digestion, termed 'bioaccessibility'; (2) uptake by the enterocyte; (3) enterocyte transport and packaging into the chylomicrons for secretion into the lymph. Several dietary factors have been shown to affect these key steps in xanthophyll absorption, e.g. the food matrix ${ }^{(9)}$ and the interactions between xanthophylls and other food components like fibre, lipids, phytosterols

Abbreviations: BHT, butylated hydroxytoluene; DMEM, Dulbecco's modified Eagle's medium; FA, fatty acid; SU.VI.MAX, Supplémentation en Vitamines et Minéraux AntioXydants.

*Corresponding author: P. Borel, fax +334917821 01, email patrick.borel@univ-amu.fr 
and other carotenoids ${ }^{(10-12)}$. Among all these dietary factors, it is assumed that co-ingestion of xanthophylls with dietary lipids has the greatest positive impact on xanthophyll absorption. This assumption is supported by the fact that the absorption efficiency of xanthophylls increases when the amount of fat in the diet increases ${ }^{(13,14)}$. Two studies have further suggested that the type of fatty acid (FA) that constitutes the oil TAG can affect xanthophyll absorption. First, a study in rats has shown that plasma lutein and zeaxanthin concentrations were markedly higher when test meals contained olive oil (rich in MUFA) compared with sunflower and groundnut oils, which are rich in PUFA $^{(15)}$. Second, an in vitro study has shown that xanthophyll bioavailability was higher in the presence of coconut oil, which is rich in SFA, than in presence of rapeseed oil, which is rich in unsaturated $F_{A}^{(16)}$. Nevertheless, natural dietary fat can contain fat-soluble micronutrients, e.g. vitamin E, $\beta$-carotene and phytosterols, and it is not known whether the observed effects were due to these micronutrients or to the different FA compositions of the sources of TAG. Thus, the mechanism(s) that can explain the effect of the source of dietary fat on xanthophyll bioavailability remains to be elucidated.

We performed the present work to allow for a firm statement on the effect of natural dietary fat on lutein/zeaxanthin bioavailability, to identify the mechanism(s) involved and to assess the effect of this dietary factor on long-term xanthophyll status in human subjects. We used three complementary models to attain our objectives: an in vitro digestion model to study bioaccessibility, Caco- 2 cell monolayers to study uptake efficiency and orally administered rats to study in vivo bioavailability. We also determined, in a cohort of 622 subjects who took part in the Supplémentation en Vitamines et Minéraux AntioXydants (SU.VI.MAX) study, whether SFA intake modulated the expected association between blood lutein and zeaxanthin concentrations and fruit and vegetable intake.

\section{Materials and methods}

\section{Chemicals}

Lutein, zeaxanthin and echinenone ( $>95 \%$ pure) were generously provided by DSM Limited (formerly F. Hoffmann-La Roche). Neoxanthin (97\% pure) was purchased from Carotenature. Porcine pepsin, porcine pancreatin, porcine bile extract, butylated hydroxytoluene (BHT), palm oil (refined) and fish oil (from menhaden) were purchased from SigmaAldrich. Potatoes, minced beef (with $5 \%$ of fat), olive oil, sunflower oil and butter were purchased from a local supermarket. Canned sweetcorn and frozen chopped spinach were provided by Bonduelle.

\section{Preparation of meals used in in vitro digestion experiments}

Meal composition is given in Table 1. Potatoes and minced beef were included in the meals to align macronutrient (carbohydrate, lipid and protein) proportions to current RDA. Indeed, as macronutrients can affect carotenoid bioavailability, it was important to use a meal that mimics the proportions
Table 1. Composition of meals used in in vitro digestion experiments

\begin{tabular}{|c|c|c|}
\hline Component & Amount (g) & Preparation \\
\hline Puréed potatoes & $6 \cdot 7$ & Boiled \\
\hline Minced beef meat ( $5 \%$ fat) & $1 \cdot 2$ & Fried \\
\hline Dietary fat* or TAG mixture & 0.2 & Incorporation of $1 \% \mathrm{BHT}$ \\
\hline Canned sweetcorn† & $4 \cdot 0$ & $\begin{array}{l}\text { Masticated by adults } \\
\text { volunteers }\end{array}$ \\
\hline Frozen chopped spinach $†$ & $4 \cdot 0$ & $\begin{array}{l}\text { Cooked with microwave } \\
\text { oven }\end{array}$ \\
\hline
\end{tabular}

BHT, butylated hydroxytoluene.

* The studied dietary fats were butter, palm, olive, sunflower and fish oils. For the butter condition, the amount of butter added was calculated to provide $0.2 \mathrm{~g}$ of fat. †Digestion experiments contained either sweetcorn or chopped spinach.

of each macronutrient found in regular meals. The test meal without the xanthophyll source contained $53.6 \%$ energy as carbohydrates, $28.4 \%$ as fat and $18.0 \%$ as proteins. These proportions are close to the US dietary reference intakes (DRI), i.e. $45-65 \%$ carbohydrates, $20-35 \%$ fat and $10-35 \%$ proteins. Potatoes were boiled in tap water, peeled and hand puréed. Meat was medium fried in a frying pan without added fat. Potato purée and fried meat were divided into aliquots and frozen at $-20^{\circ} \mathrm{C}$. The sweetcorn source that was used in all experiments was preliminarily masticated by eight adult volunteers from the laboratory to mimic at best the in vivo conditions. Then, it was divided into aliquots and frozen at $-20^{\circ} \mathrm{C}$. The chopped spinach $(30 \mathrm{~g}$ frozen) was cooked in a microwave oven, for $1 \mathrm{~min}$ at $750 \mathrm{~W}$, on the days of the experiments. Just before the experiments, BHT was added into the different sources of dietary fat (1\% in weight), either natural dietary fats or pure TAG mixtures (Table 2), in order to prevent oxidation that could degrade carotenoids during the in vitro digestion experiments. To accurately assess the amount of carotenoids present in the meals, the meal components were mixed with $32 \mathrm{ml}$ of $\mathrm{NaCl}$ $0.9 \%$ with an ultra-turrax disperser (Ika) to obtain a homogeneous purée. Aliquots of this purée were stored at $-80^{\circ} \mathrm{C}$ under a $\mathrm{N}_{2}$ atmosphere until carotenoid analysis by HPLC (see Carotenoid analysis section).

\section{In vitro digestion experiments}

The in vitro digestion model was adapted from Reboul et al. ${ }^{(9)}$ with minor modifications. Meal components, which contained either masticated sweetcorn or chopped spinach, were mixed with $32 \mathrm{ml}$ of $0.9 \% \mathrm{NaCl}$ (ultra-turrax disperser (Ika), $30 \mathrm{~s}$ at $6000 \mathrm{rpm}$ ), and $2 \mathrm{ml}$ of artificial saliva was added to the mixture ${ }^{(17)}$. The samples were incubated for $10 \mathrm{~min}$ in a shaking water-bath at $37^{\circ} \mathrm{C}$. The $\mathrm{pH}$ was then adjusted to $4 \pm 0.02$ with about $500 \mu \mathrm{l}$ of $1 \mathrm{M}-\mathrm{HCl}$. Then, $2 \mathrm{ml}$ of porcine pepsin $(40 \mathrm{mg} / \mathrm{ml}$ in $0.1 \mathrm{M}-\mathrm{HCl})$ was added and the mixture was incubated at $37^{\circ} \mathrm{C}$ in a shaking water-bath for $30 \mathrm{~min}$ to mimic the gastric phase of digestion. The $\mathrm{pH}$ of the partially digested mixture was raised to $6 \pm 0.02$ by adding about $800 \mu \mathrm{l}$ of $0.9 \mathrm{M}$-sodium bicarbonate $(\mathrm{pH}$ 9-10). Then, $9 \mathrm{ml}$ of a mixture of porcine bile extract and pancreatin (containing $2 \mathrm{mg} / \mathrm{ml}$ pancreatin and $12 \mathrm{mg} / \mathrm{ml}$ bile extract in 
Table 2. Fatty acid composition of dietary fat and TAG mixtures (percentage in weight) used in in vitro digestion experiments

\begin{tabular}{|c|c|c|c|c|c|c|c|c|c|c|}
\hline & \multicolumn{2}{|c|}{ Butter } & \multicolumn{2}{|c|}{ Palm oil } & \multicolumn{2}{|c|}{ Olive oil } & \multicolumn{2}{|c|}{ Sunflower oil } & \multicolumn{2}{|c|}{ Fish oil } \\
\hline & Fat & TAG mix & Fat & TAG mix & Fat & TAG mix & Fat & TAG mix & Fat & TAG mix \\
\hline SCFA $^{*}$ & $25 \cdot 4$ & 26 & 0.8 & & & & & & 9 & \\
\hline Lauric acid & $3.5 \dagger$ & $26 \dagger$ & & & & & & & & \\
\hline Myristic acid & $10 t$ & & $0.8 \dagger$ & & & & & & 9 & \\
\hline SFA & 42.5 & 42 & 46.4 & 47 & $14 \cdot 3$ & 14 & $10 \cdot 6$ & 10 & $22 \cdot 7$ & 31 \\
\hline Palmitic acid & $29 \cdot 1 \dagger$ & $42 \dagger$ & $41.8 \dagger$ & $47 \dagger$ & $10.8 \dagger$ & $14 \dagger$ & $6.4 \dagger$ & $10 \dagger$ & $18.9 \dagger$ & $31 \dagger$ \\
\hline Stearic acid & $13.4 \dagger$ & & $4.5 t$ & & $3.4 \dagger$ & & $4.2 \dagger$ & & $3.7 \dagger$ & \\
\hline MUFA & 28.0 & 28 & 42.5 & 43 & 79.0 & 80 & $27 \cdot 2$ & 26 & 24.6 & 25 \\
\hline Palmitoleic acid & $1.3 \dagger$ & & $0.1+$ & & $0.9 \dagger$ & & $6.4 \dagger$ & & $13.1+$ & $25 t$ \\
\hline Oleic acid & $26 \cdot 7 \dagger$ & $28 \dagger$ & $42.4 \dagger$ & $43 \dagger$ & $78.1 \dagger$ & $80 \dagger$ & $26 \cdot 2 \dagger$ & $26 \dagger$ & $11.5 \dagger$ & $25 \dagger$ \\
\hline PUFA & $4 \cdot 1$ & 4 & $10 \cdot 3$ & 10 & $6 \cdot 7$ & 6 & $63 \cdot 1$ & 64 & $43 \cdot 8$ & 44 \\
\hline Linoleic acid & $3.7 \dagger$ & $4 \dagger$ & $10.1 \dagger$ & $10 \dagger$ & $6 \cdot 1 \dagger$ & $6 \dagger$ & $62.3 \dagger$ & $64 \dagger$ & $1.9 \dagger$ & $0 \dagger$ \\
\hline EPA & & & & & & & & & $16 \cdot 1 \dagger$ & $22 \dagger$ \\
\hline $\mathrm{DHA}$ & & & & & & & & & $17 \cdot 1 \dagger$ & $22 \dagger$ \\
\hline
\end{tabular}

* SCFA: fatty acids containing from four to fourteen carbons. TAG mixes were achieved with trilaurin, tripalmitin, triolein, trilinolein, trieicosapentaenoin and tridocosahexaenoin. Butylated hydroxytoluene (1\% in weight) was added into the mixtures in order to preserve the xanthophylls from degradation (oxidation). The fatty acid composition was determined by GC according to the method described previously ${ }^{(41)}$.

† Main individual fatty acids in each fatty acid family.

$100 \mathrm{~mm}$-trisodium citrate ( $\mathrm{pH}$ 6.0)) and $4 \mathrm{ml}$ of porcine bile extract at $0 \cdot 1 \mathrm{~g} / \mathrm{ml}$ were added. Samples were further incubated in a shaking water-bath at $37^{\circ} \mathrm{C}$ for $30 \mathrm{~min}$ to mimic digestion within the duodenum.

The aqueous fraction, which contains the mixed micelles that were produced upon in vitro digestion, was separated from oil droplets and food particles by centrifugation $(2200 \boldsymbol{g}$ for $1 \mathrm{~h}$ at $10^{\circ} \mathrm{C}$ ). The aqueous fraction was collected and was passed through 0.8 and $0.22 \mu \mathrm{m}$ filters (Millipore) in order to discard remaining small food particles and to obtain a clear solution of mixed micelles. Aliquots were stored at $-80^{\circ} \mathrm{C}$ under a $\mathrm{N}_{2}$ atmosphere until carotenoid analysis.

\section{Experiments on uptake efficiency of micellar xanthophylls by intestinal cells}

Caco-2 clone TC-7 cells ${ }^{(18,19)}$ were a gift from Dr M. Rousset (UMR_S 872). Cells were cultured in the presence of Dulbecco's modified Eagle's medium (DMEM) supplemented with $20 \%$ heat-inactivated fetal bovine serum, $1 \%$ nonessential amino acid and $1 \%$ antibiotics (complete medium). Cells were incubated at $37^{\circ} \mathrm{C}$ in a humidified atmosphere of air $-\mathrm{CO}_{2}(90: 10, \mathrm{v} / \mathrm{v})$ and the medium was changed every $48 \mathrm{~h}$, as described previously ${ }^{(20)}$.

For each experiment, cells were seeded at a density of $25 \times 10^{4}$ cells/well and grown on transwell membrane (sixwell plate, $1 \mu \mathrm{m}$ pore size polycarbonate membrane; Becton Dickinson), and were cultured as described previously ${ }^{(20)}$.

At the beginning of each experiment, cell monolayers were washed twice with $1 \mathrm{ml}$ of PBS. The apical side of the cell monolayers received $1 \mathrm{ml}$ of mixed micelles that came from the in vitro digestion experiments (diluted at $1 / 3$ in DMEM). Cell monolayers were incubated at $37^{\circ} \mathrm{C}$ for $2 \mathrm{~h} 30 \mathrm{~min}$. The incubation time has been chosen after a preliminary experiment to obtain sufficient amounts of absorbed carotenoids for accurate measurements. After the incubation period, media from each side of the membrane were harvested. Cell monolayers were washed twice with $1 \mathrm{ml}$ of ice-cold PBS, scraped and collected in $500 \mu \mathrm{l}$ of PBS. All samples were stored at $-80^{\circ} \mathrm{C}$ under a $\mathrm{N}_{2}$ atmosphere until carotenoid analysis.

\section{Determination of spinach lutein bioavailability in rats}

The animal model chosen to study lutein bioavailability was multiple-dose oral administration over $3 \mathrm{~d}$ in young male albino Wistar rats (6 weeks old) of normal weight (about $200 \mathrm{~g}$ ). Experiments were conducted according to animal ethics policies and were approved by the experimental animal ethics committee of Aix-Marseille University. All rats were fed the same standard diet ad libitum throughout the experiment. The rats were randomly divided into three groups ( $n 10$ per group). The different groups of rats were orally administered with $1 \mathrm{ml}$ of spinach/dietary fat emulsion (spinach-dietary fat-water; $1: 1: 1$ by weight for $1 \mathrm{ml}$ ). We used spinach instead of sweetcorn as the source of xanthophylls in the present animal study because it was not possible to have homogeneous administration with sweetcorn, which contains a hull that is difficult to grind homogeneously. Nevertheless, it should be reminded that there was no effect of the vegetable matrix on xanthophyll bioaccessibility.

The dietary fats used in the present study were butter, olive and fish oils. Oral administration was performed every evening for three consecutive days. At $16 \mathrm{~h}$ after the last administration, an intra-cardiac venepuncture was performed on anaesthetised rats. The plasma samples were obtained after centrifugation $\left(500 \mathrm{~g}, 10 \mathrm{~min}, 10^{\circ} \mathrm{C}\right)$ of blood samples and stored at $-80^{\circ} \mathrm{C}$ until carotenoid analysis.

Before the protocol, a blood sample of eight rats randomly chosen was collected by intra-cardiac venepuncture to check baseline plasma lutein concentration, which was expected and found to be null because there was no lutein in the rat diet.

\section{Subject sample}

In order to assess whether long-term FA consumption, by affecting xanthophyll bioavailability, may in turn affect blood concentrations of xanthophylls in human subjects, we 
decided to determine whether FA intake modulated the expected association between blood lutein and zeaxanthin concentrations and fruit and vegetable intake. To do that, we used data of the 'SU.VI.MAX' study. This study was designed to test the benefits of a multivitamin-mineral supplementation on cancer and CVD in France ${ }^{(21)}$. Eligibility criteria were lack of disease likely to hinder active participation or threatened 5-year survival; lack of previous regular supplementation with any of the vitamins or minerals in the supplement provided; and absence of extreme beliefs or behaviour regarding diet. Further exclusion criteria after an examination by a physician were: suffering from cancer; diabetes; severe kidney, liver, lung diseases or CVD; mental or physical disability; neurological and psychiatric diseases; and inborn errors of metabolism. In 2007-09, a total of 6850 subjects who agreed to participate in a post-supplementation follow-up were included in the SU.VI.MAX 2 study, which sought to investigate the impact of nutrition on quality of ageing. Clinical and biological measurements were assessed. A subsample of 622 subjects was then selected, for whom plasma carotenoids were measured (i.e. the sample studied in the present work). The characteristics of this subsample are given in detail in previous publications ${ }^{(22,23)}$. During the SU.VI.MAX 2 study, participants were asked to complete a validated FFQ including 240 items $^{(23)}$. Nutrient intakes were estimated from FFQ using a food composition table $\mathrm{e}^{(24)}$. Socio-demogaphic data and lifestyles were self-reported in questionnaires.

\section{Xanthophyll analysis}

Xanthophylls were extracted as described previously ${ }^{(20)}$. The procedure was as follows: $500 \mu \mathrm{l}$ of sample were added to $500 \mu \mathrm{l}$ of ethanol-containing internal standard (echinenone).

The mixture was extracted twice with two volumes of hexane. The hexane phases obtained after centrifugation $\left(500 \mathrm{~g}, 5 \mathrm{~min}, 10^{\circ} \mathrm{C}\right.$ ) were evaporated to dryness under $\mathrm{N}_{2}$, and the dried extract was dissolved in $200 \mu \mathrm{l}$ of methanoldichloromethane $(65: 35, \mathrm{v} / \mathrm{v})$. A final volume of $50 \mu \mathrm{l}$ for the purée sample, $100 \mu \mathrm{l}$ for the mixed micelle sample, $160 \mu \mathrm{l}$ for cellular and rat plasma samples and $20 \mu \mathrm{l}$ for the human plasma sample were used for HPLC analysis.

Xanthophylls were separated as recently described ${ }^{(25)}$. The HPLC system comprised a Dionex separation module (P680 HPLC Pump and ASI-100 Automated Sample Injector) and a Dionex UVD340U photodiode array detector (Dionex SA). Analyses were performed on a $250 \times 4.6 \mathrm{~nm} \mathrm{RP} \mathrm{C30,} 5 \mu \mathrm{m}$ YMC column coupled with a $20 \times 4.6 \mathrm{~mm}$ inner diameter C18, $5 \mu \mathrm{m}$ Zorbax guard column (Interchim) and kept at $35^{\circ} \mathrm{C}$. Carotenoids were detected at $450 \mathrm{~nm}$ and identified by retention time and spectral analysis $(300-500 \mathrm{~nm}$ ) compared with pure standards of xanthophylls and echinenone. Quantification was performed using Chromeleon software (version 6.8.SR7, Dionex) by comparing peak area with standard reference curves $(1-100 \mu \mathrm{g} / \mathrm{ml})$ and corrected by extraction efficiency based on the recovery of internal standard (95\% in mean).

\section{Measurement of mixed micelle size and zeta potential}

The intensity-weighted mean hydrodynamic radius and the zeta potential (which reflects the electric potential on the surface of particles) of the mixed micelles were determined by photon correlation spectroscopy at $25^{\circ} \mathrm{C}$ (Zetasizer Nano $\mathrm{Zs}$, Malvern Instruments) directly following the preparation of mixed micelles. The samples were stored at room temperature prior to analysis.

\section{Calculation and statistical analysis}

Bioaccessibility was defined as the percentage of xanthophylls recovered in the micellar fraction after in vitro digestion, in relation to the amount of xanthophylls measured in the digestive medium just before the addition of artificial saliva. Cellular uptake efficiency was defined as the percentage of carotenoids, added in micelles in the apical chamber of the cell culture, which was recovered in the scraped cells. We defined the in vitro bioavailability of xanthophylls as the product of their bioaccessibility by their cellular uptake efficiency. The degree of FA unsaturation of TAG was calculated as the sum of the molar percentage multiplied by the number of double bond content of each FA in the fat studied. Results are expressed as means with their standard errors. Mean values obtained in both the in vitro experiments and in the rat study were compared by the non-parametric Kruskal-Wallis test, followed by the Mann-Whitney $U$ test when the Kruskal-Wallis test found significant $(P<0.05)$ differences. Correlations between data obtained in the in vitro experiments were tested using non-parametric regression analyses (Spearman's rank tests). In the human study, the association between fasting plasma xanthophyll concentrations and fruit and vegetable intakes were estimated through covariance analysis according to SFA intake. All dietary and nutrient intake were adjusted for energy intake using the residual method ${ }^{(26)}$. Sex-specific tertiles of fruit and vegetable consumption were calculated. Models were adjusted for age, sex, education, tobacco status, supplementation group during the trial phase, energy and alcohol intake, BMI, season of biological data collection and cholesterolaemia $^{(27-29)}$. Probability values of less than 0.05 were considered significant. Statistical analyses were performed using Statview and SAS softwares (SAS Institute).

\section{Results}

Size and zeta potential of mixed micelles recovered after in vitro digestion of meals containing sweetcorn and different types of dietary fat

The size and zeta potential of mixed micelles produced during the in vitro digestions were modified by the type of dietary fat present in the meal (Table 3). More precisely, the presence of butter or palm oil induced a mean radius of mixed micelles significantly smaller than the presence of sunflower oil $(-6 \%)$ and olive or fish oils $(-9 \%)$. The zeta potential of mixed micelles was always negative and the electrical charge of mixed micelles from butter and palm oil was significantly lower than those from 
Table 3. Size and zeta potential of mixed micelles* recovered after in vitro digestion of meals containing different dietary fats

(Mean values with their standard errors of five independent experiments)

\begin{tabular}{|c|c|c|c|c|}
\hline & \multicolumn{2}{|c|}{$\begin{array}{l}\text { Size (mean } \\
\text { radius, } \mathrm{nm} \text { ) }\end{array}$} & \multicolumn{2}{|c|}{ Zeta potential $(\mathrm{mV})$} \\
\hline & Mean & SEM & Mean & SEM \\
\hline Butter & $2 \cdot 85^{\mathrm{a}}$ & 0.07 & $-20 \cdot 00^{\mathrm{a}, \mathrm{c}}$ & 0.62 \\
\hline Palm oil & $2 \cdot 88^{\mathrm{a}, \mathrm{c}}$ & 0.04 & $-20 \cdot 14^{a}$ & 0.41 \\
\hline Olive oil & $3 \cdot 12^{b}$ & 0.07 & $-20 \cdot 34^{a, b}$ & 1.09 \\
\hline Sunflower oil & $3.05^{\mathrm{b}}$ & 0.05 & $-17.74^{\mathrm{b}}$ & 0.32 \\
\hline Fish oil & $3 \cdot 12^{b, c}$ & 0.10 & $-17 \cdot 82^{\mathrm{b}, \mathrm{c}}$ & 0.75 \\
\hline
\end{tabular}

${ }^{a, b, c}$ Mean values within a column with unlike superscript letters are significantly different $(P<0.05)$.

* The intensity-weighted mean radius (size) and the zeta potential of the lipid particles of micellar fraction were determined by photon correlation spectroscopy at $25^{\circ} \mathrm{C}$.

sunflower and fish oils ( $12 \%$ on average). The size and zeta potential of mixed micelles were positively correlated $(\rho=0 \cdot 43, P=0 \cdot 033)$.

\section{Effect of source and type of TAG fatty acid on xanthophyll bioaccessibility}

It is worth mentioning that when no BHT (2,6-di-tert-butyl4-methylphenol, a commonly used antioxidant) was incorporated in the tested fat sources (see the Materials and methods section) in the in vitro digestions, we observed a significant degradation of xanthophylls, particularly in the presence of fish oil (source of PUFA very sensitive to oxidation; data not shown). Because this oxidation generated a confounding variable, which did not allow to correctly assess the effect of the type of fat on xanthophyll bioaccessibility, we added BHT in all subsequent digestion experiments. A control study (results not shown) showed that in the presence of BHT, xanthophylls and FA were not degraded.

\section{Effect of source of natural dietary fat on sweetcorn xanthophyll bioaccessibility}

The first noteworthy observation made was that the bioaccessibility of zeaxanthin, i.e. the proportion of the sweetcorn zeaxanthin that was incorporated into mixed micelles, was systematically lower (about 10\% on average) than that of lutein (Fig. 1). The second observation was that the bioaccessibility of the xanthophylls was different when the source of dietary fat incorporated in the test meal was different. More precisely, xanthophyll bioaccessibility was significantly greater with butter, palm and sunflower oils than with olive and fish oils ( $20 \%$ on average for lutein and $23 \%$ for zeaxanthin). Besides, as displayed in Fig. 2, there was a significant negative correlation between xanthophyll bioaccessibility and mixed micelle size $(\rho=-0 \cdot 40, P=0.048$ for lutein and $\rho=-0 \cdot 46, P=0.022$ for zeaxanthin). Conversely, there was no significant correlation between xanthophyll bioaccessibility and mixed micelle zeta potential (data not shown).

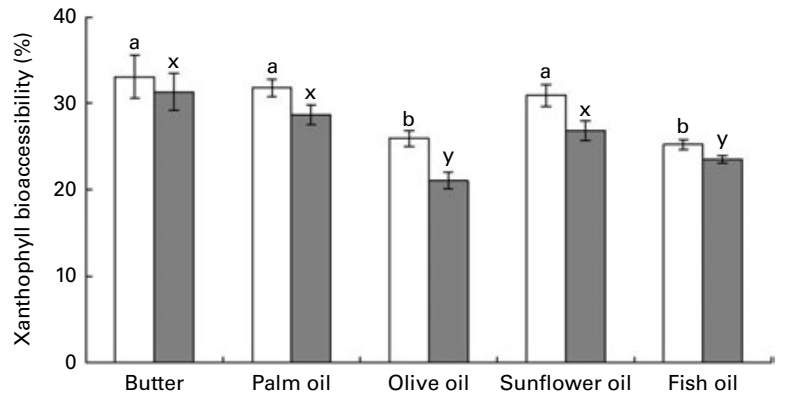

Fig. 1. Effect of the type of dietary fat on the bioaccessibility of lutein ( $\square$ ) and zeaxanthin $(\square)$ from sweetcorn. Bioaccessibility refers to the percentage of sweetcorn xanthophylls found in the micelle fraction after the in vitro digestion experiments. Values are means of five independent experiments for lutein and zeaxanthin, with their standard errors represented by vertical bars. ${ }^{a, b}$ Mean values with unlike letters were significantly different $(P<0.05)$. ${ }^{x, y}$ Mean values with unlike letters were significantly different $(P<0.05)$.

Effect of pure TAG mixtures that mimicked the fatty acid compositions of natural fat on sweetcorn xanthophyll bioaccessibility

Natural dietary fats contain fat-soluble microconstituents, e.g. phytosterols (sunflower oil), polyphenol (olive oil) and $\beta$ carotene (butter), which can affect xanthophyll bioaccessibility. Therefore, to eliminate these interferences and to be able to accurately assess the effect of the type of TAG FA, we measured xanthophyll bioaccessibility when sweetcorn was digested with mixtures of pure TAG that mimicked the FA compositions of the studied natural dietary fat sources (Table 2). Results showed that
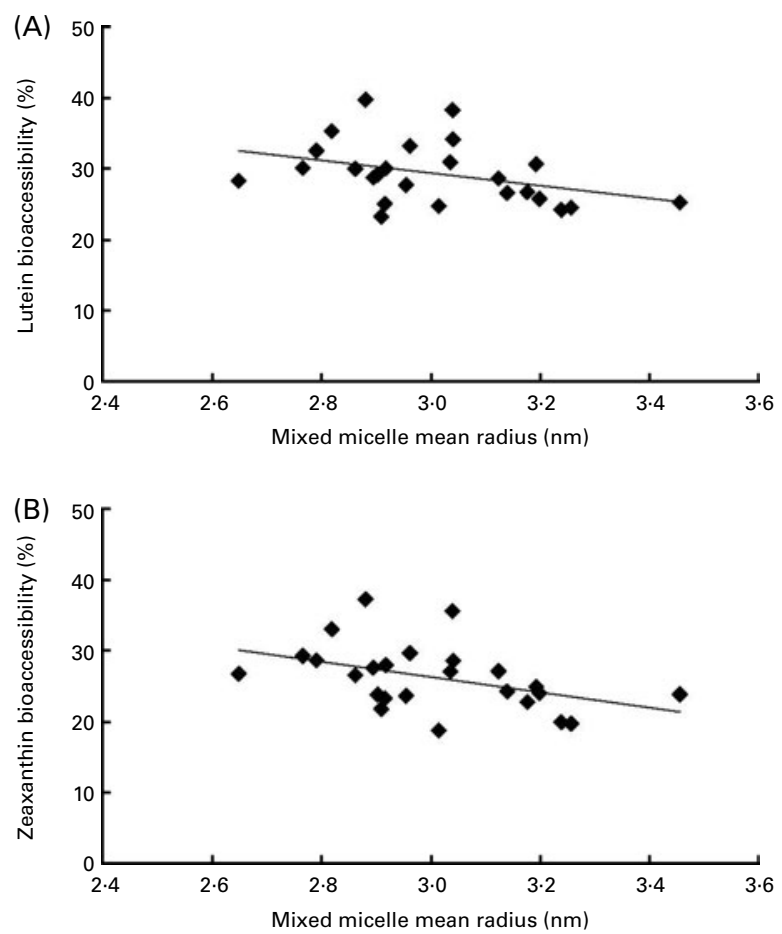

Fig. 2. Relationship between the size of mixed micelles found in the medium of the in vitro digestion experiments and the bioaccessibility of sweetcorn xanthophylls. (A) Correlation (Spearman's rank test) between the size of mixed micelles and lutein bioaccessibility $(\rho=-0.404, P=0.048)$. (B) Correlation between the size of mixed micelles and zeaxanthin bioaccessibility $(\rho=-0.466, P=0.022)$. 
xanthophyll bioaccessibility was significantly higher with the butter-like TAG mix than with the palm oil-like TAG mix and olive oil-like TAG mix (data not shown). Finally, the sunflower oil-like TAG and fish oil-like TAG mixes exhibited comparable and significantly lower xanthophyll bioaccessibility, as compared with the other TAG mixes. Correlation analyses showed a negative relationship between the degree of FA unsaturation of a TAG mix and the bioaccessibility of lutein $(\rho=-0 \cdot 80$, $P=0.0004)$ and zeaxanthin $(\rho=-0.77, P=0.0008)$. They also showed a negative correlation between the TAG FA chain length and lutein $(\rho=-0.80, P=0.0004)$ and zeaxanthin ( $\rho=-0.77, P=0.0008$ ) bioaccessibility. Finally, we found that mixed micelle size, which was significantly affected by the type of a TAG mix added in the in vitro digestion experiments (data not shown), was inversely related to xanthophyll bioaccessibility $(\rho=-0.404, P=0.0479$ for lutein and $\rho=-0.466$, $P=0.0223$ for zeaxanthin).

\section{Effect of source of natural dietary fat on spinach xanthophyll bioaccessibility}

In order to establish that the observed effects of dietary fat on xanthophyll bioaccessibility were not specific to the sweetcorn matrix, we measured the bioaccessibility of the main spinach xanthophylls, i.e. lutein and neoxanthin, under the same experimental conditions. Results showed once again that butter led to a higher bioaccessibility of the xanthophylls than olive, sunflower and fish oils (Table S1, available online). The positive effect of butter on xanthophyll bioaccessibility was therefore similar for the three studied xanthophylls, i.e. lutein, zeaxanthin and neoxanthin, either when they were in sweetcorn or in spinach.

\section{Effect of source and type of TAG fatty acids on xanthophyll uptake by intestinal Caco-2 cells}

Cellular uptake efficiency of micellarised zeaxanthin was systematically higher than that of micellarised lutein (about 19\% on average). Furthermore, uptake efficiency of sweetcorn xanthophylls was significantly affected by the source of dietary fat incorporated into the meal (Fig. 3). More precisely, sweetcorn xanthophyll uptake efficiency was significantly higher in the presence of palm and olive oils than in the presence of sunflower and fish oils (24\% in mean for lutein and $22 \%$ for zeaxanthin). Butter showed a high/intermediate uptake efficiency. There was no significant correlation between xanthophyll cellular uptake level and mixed micelle size (data not shown).

We performed similar cellular uptake experiments with mixed micelles that were issued from digestion of mixtures of pure TAG in order to avoid the potential effects of fat-soluble microconstituents present in natural fats. The data obtained showed no significant effect of the nature of TAG mix on xanthophyll uptake efficiency by Caco-2 cells (data not shown).

Finally, lutein- and neoxanthin-rich micelles, as issued upon in vitro digestion of spinach with different sources of dietary fat, were taken up with a similar efficiency, whatever the fat used: butter, palm or fish oil (Table S2, available online).

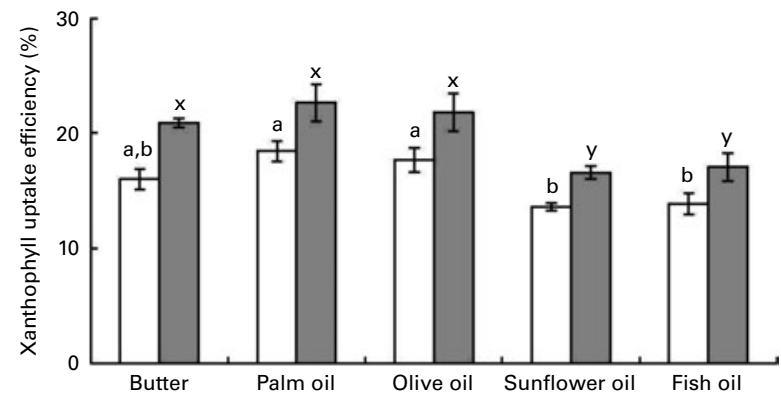

Fig. 3. Effect of the type of fat used in the in vitro digestion experiments on the uptake efficiency of sweetcorn xanthophylls by Caco-2 cell monolayers. Mixed micelles recovered after digestion of meals containing sweetcorn and different dietary fats were added on Caco-2 monolayers to measure xanthophyll cellular uptake. Uptake efficiency refers to the percentage of added micellarised sweetcorn xanthophylls recovered in the Caco- 2 cells after $2 \mathrm{~h} 30 \mathrm{~min}$ incubation. Values are means of five independent experiments for lutein $(\square)$ and zeaxanthin $(\square)$, with their standard errors represented by vertical bars. ${ }_{\mathrm{a}, \mathrm{b}}$ Mean values with unlike letters were significantly different $(P<0.05)$. ${ }^{\mathrm{x}, \mathrm{y}}$ Mean values with unlike letters were significantly different $(P<0.05)$.

\section{Effect of source and type of TAG fatty acids on in vitro bioavailability of xanthophylls}

The data obtained after calculation of the in vitro bioavailability (Fig. 4) showed: (1) that sweetcorn zeaxanthin bioavailability was generally higher (about $10 \%$ on average) than that of sweetcorn lutein and (2) that the bioavailability of lutein and zeaxanthin was significantly greater in the presence of butter and palm oil than in the presence of the other unsaturated fats. More precisely, other fats showed a lower bioavailability of lutein, as compared with butter and palm oil ( $-21 \%$ for olive oil, $-28 \%$ for sunflower oil and $-40 \%$ for fish oil) and of zeaxanthin ( $-30 \%$ for olive oil, $-32 \%$ for sunflower oil and $-39 \%$ for fish oil).

The data of in vitro bioavailability with the mixtures of pure TAG are presented in Fig. 5. The butter-like TAG mix induced a significantly higher in vitro bioavailability of xanthophylls, as compared with the other TAG mixtures. Conversely, the fish oil-like TAG mix elicited a significantly lower in vitro xanthophyll bioavailability.

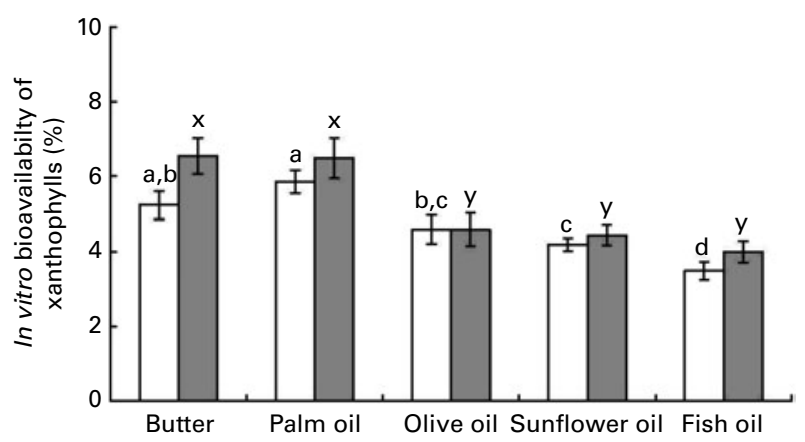

Fig. 4. Effect of the type of dietary fat on the in vitro bioavailability of sweetcorn xanthophylls. In vitro bioavailability refers to the percentage of sweetcorn xanthophylls transferred from the food matrix to the Caco-2 cells. It was calculated as the product of bioaccessibility (\%) by uptake efficiency (\%). Values are means of five independent experiments for lutein ( $\square$ ) and zeaxanthin ( $\square$ ), with their standard errors represented by vertical bars. ${ }^{a, b, c, d}$ Mean values with unlike letters were significantly different $(P<0 \cdot 05) .{ }^{\mathrm{x}, \mathrm{y}}$ Mean values with unlike letters were significantly different $(P<0.05)$. 


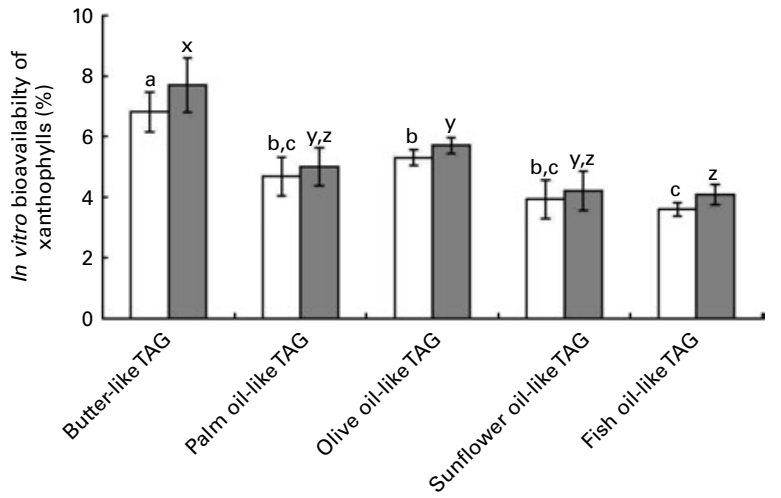

Fig. 5. Effect of different TAG mixtures on the in vitro bioavailability of sweetcorn xanthophylls. The mixtures of TAG were elaborated to mimic the fatty acids composition of the studied dietary fat sources. In vitro bioavailability refers to the percentage of sweetcorn xanthophylls transferred from the food matrix to the Caco-2 cells. It was calculated as the product of bioaccessibility (\%) by uptake efficiency (\%). Values are means of five independent experiments for lutein $(\square)$ and zeaxanthin $(\square)$, with their standard errors represented by vertical bars. ${ }^{a, b, c}$ Mean values with unlike letters were significantly different $(P<0.05) .{ }^{x, y, z}$ Mean values with unlike letters were significantly different $(P<0.05)$.

Moreover, we calculated in vitro bioavailability after the digestion and cellular uptake of spinach xanthophylls. The results obtained were in agreement with those obtained with the sweetcorn matrix, with a significantly higher in vitro bioavailability of the xanthophylls with butter as compared with olive, sunflower and fish oils (Table S3, available online).

\section{Effect of source of dietary fat on spinach lutein bioavailability in rats}

In order to check whether the in vitro data could predict in vivo bioavailability, we determined the effect of three dietary fats on lutein bioavailability in an in vivo model. Adult rats were orally administered with a xanthophyll source (spinach) together with three different TAG emulsions made with butter, olive or fish oil for three consecutive days. The butter emulsion led to a significantly higher fasting plasma concentration of lutein than the olive oil emulsion, and even more markedly than the fish oil emulsion (Fig. 6).

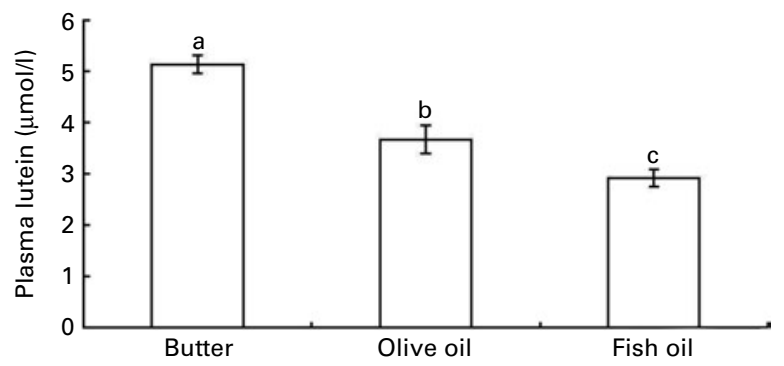

Fig. 6. Effect of different types of dietary fat used in oral administration experiments in rats on fasting plasma lutein concentration. Rats ( $n 10$ per group) were orally administered for three consecutive days with $1 \mathrm{ml}$ spinach/dietary fat emulsions (for more details, see the Materials and methods section). Values are means, with their standard errors represented by vertical bars. ${ }^{a, b, c}$ Mean values with unlike letters are significantly different $(P<0.05)$
Effect of dietary intake of SFA on associations between plasma xanthophyll concentrations and fruit and vegetable intake

As shown in Table 4 , both lutein \pm zeaxanthin and $\beta$-cryptoxanthin plasma concentrations were significantly positively associated with fruit and vegetable intake among subjects with a low $(<34-37 \mathrm{~g} / \mathrm{d})$ dietary intake of SFA. Conversely, this association was not observed for lutein \pm zeaxanthin in subjects with a high $(>34-37 \mathrm{~g} / \mathrm{d})$ dietary intake of SFA.

\section{Discussion}

In order to understand how the type of FA that constitutes dietary TAG can affect xanthophyll absorption efficiency, we used two complementary models to study the key steps of xanthophyll absorption: an in vitro digestion model, to study carotenoid bioaccessibility, and intestinal Caco-2 monolayers, to study uptake by intestinal cells. In vivo data in rats and association studies using data from a human study were obtained to substantiate these in vitro observations.

Data obtained with the in vitro digestion model showed that the dietary fat sources that had the higher proportions of SFA, i.e. butter and palm oil, led to a higher bioaccessibility of the xanthophylls than the fat sources that were rich in long-chain MUFA and PUFA. This result did not depend on the vegetable matrix in which the xanthophylls were incorporated because comparable data were obtained with either sweetcorn or spinach. This finding is in agreement with previous studies showing that lutein and zeaxanthin were more bioaccessible in the presence of coconut or peanut oil (rich in SFA) than in the presence of rapeseed oil (rich in unsaturated FA) ${ }^{(16,30)}$. The present result raises the following question: does the effect of the source of dietary fat on xanthophyll bioaccessibility depends on its FA composition or does it depend on the presence of fat-soluble microconstituents in some sources of dietary fats (e.g. phytosterols and vitamin $\mathrm{E}$ in sunflower oil, $\beta$-carotene in butter $)^{(31,32)}$ ? To answer this question, we performed digestion experiments in which we replaced the natural sources of dietary TAG by mixtures of pure TAG that mimicked the FA composition of the natural studied fats. These experiments provided similar findings to those using natural TAG sources, i.e. butter- and palm oil-like TAG mixtures led to a higher xanthophyll bioaccessibility than sunflower and fish oils-like TAG. We therefore conclude that the microconstituents present in the studied natural sources of dietary TAG have no major effect on xanthophyll bioaccessibility.

In order to understand the mechanism(s) involved, we determined correlations between FA characteristics and xanthophyll bioaccessibility. These calculations showed that there was a significant $(P<0.05)$ negative relationship between the degree of unsaturation of TAG FA and xanthophyll bioaccessibility. We also found a significant $(P<0.05)$ negative relationship between xanthophyll bioaccessibility and the mean length of FA present in the TAG. These observed relationships thus support the hypothesis that the physicochemical properties of TAG FA play a key role in xanthophyll bioaccessibility. To go further into the mechanism(s) involved, 
we measured the size and zeta potential of mixed micelles. Results showed that the size of the mixed micelles produced on in vitro digestion of the fats rich in SFA were lower than those produced from the fats rich in unsaturated FA. In fact, micelle mean radius was positively related $(P<0.005)$ to the degree of unsaturation of FA that constituted the fat sources. This is in agreement with a previous publication that has shown that addition of long-chain FA to bile salt solutions increased the micellar size ${ }^{(33)}$ but, to our knowledge, this is the first demonstration of an effect of the unsaturation degree of FA on mixed micelle size. However, the most interesting finding of these association studies, with regard to our research question, was the significant $(P<0.05)$ inverse relationship between micelle size and xanthophyll bioaccessibility. Indeed, the lower the unsaturation degree of $\mathrm{FA}$, the lower the size of mixed micelles and the higher their ability to solubilise the xanthophylls. To explain this finding, we hypothesise that lutein and zeaxanthin, which possess two polar groups, have these groups located at the micelle surface. As reducing the size of a fixed amount of lipid particles increases its specific surface area ${ }^{(34)}$, we hypothesise that the lower size of micelles rich in SFA increases the amount of xanthophylls that can be incorporated per amount unit of micelle lipids.

The second mechanism by which TAG FA could have an effect on xanthophyll absorption is an effect on xanthophyll uptake by intestinal cells. Nevertheless, we did not find any significant effect of the type of TAG FA on xanthophyll uptake by Caco- 2 cells when pure TAG mixtures were used. Thus, we conclude that the type of FA does not have an important effect on this step of xanthophyll absorption. However, the significant differences observed on xanthophyll uptake when mixed micelles produced on digestion of natural fats suggest that some compounds in natural fats, probably fat-soluble micronutrients, may affect xanthophyll uptake by Caco- 2 cells. This hypothesis is supported by previous publications ${ }^{(20,35)}$.

To validate the earlier in vitro findings, we performed a study in a rat model, i.e. rats that were orally administered with a mixture of spinach plus different fat sources for $3 \mathrm{~d}$. The results obtained supported well the ones obtained with the in vitro models, with a higher in vivo xanthophyll bioavailability in the presence of butter compared with unsaturated dietary fats. The significantly lower xanthophyll bioavailability observed when spinach was orally administered with fish oil, as compared with olive oil, was noteworthy given there was no significant difference between these two oils with regard to in vitro xanthophyll bioaccessibility (Fig. 1). A first hypothesis to explain this finding is that the observed difference between these two oils with regard to xanthophyll uptake by intestinal cells (Fig. 3) may have a significant effect on xanthophyll bioavailability in vivo. A second hypothesis is that the difference in plasma lutein concentration between rats orally administered with olive and fish oils was due to a partial degradation of the xanthophyll in the rat gastrointestinal tract in the presence of the highly oxidisable fish oil $\mathrm{FA}^{(36,37)}$. Indeed, we did not add BHT in the fat sources used in the rat study to be closer to dietary conditions, but we found that xanthophylls were partially degraded in the in vitro digestion experiments when no BHT was added. A third hypothesis is that, as has previously been 
reported $^{(38-40)}$, MUFA in olive oil had induced a higher secretion of chylomicrons, and thus a higher bioavailability of lutein, as compared with PUFA present in fish oil. In conclusion, we suggest that the in vivo bioavailability of xanthophylls can be negatively affected by fish oils by three mechanisms: (1) by intrinsic physical properties of fish oil FA that lead to bigger micelles that solubilise less xanthophylls, (2) by an inhibitory effect of a micronutrient present in fish oil on xanthophyll uptake by intestinal cells and (3) by the oxidation of fish oil FA in the gastrointestinal tract, which in turn may degrade a fraction of the antioxidant xanthophylls.

Overall, the results obtained with the complementary models suggest that butter, and probably palm oil, enhances xanthophyll bioavailability, as compared with olive, sunflower and fish oils. This effect is apparently due to the higher bioaccessibility of xanthophylls induced by the smallest micelles produced in the presence of SFA. However, other mechanisms are apparently involved, such as a possible inhibitory effect of some fatsoluble micronutrients, which may impair xanthophyll uptake by intestinal cells, and partial degradation of the xanthophylls in the gastrointestinal tract in the presence of highly oxidisable FA. Finally, it should be reminded that the type of TAG FA might also affect the intracellular transport of xanthophylls in the enterocyte and their packaging in the chylomicrons, mechanisms that were not assessed in the present study.

The last question was to determine whether long-term SFA consumption, by affecting xanthophyll bioavailability, may in turn affect blood concentrations of xanthophylls in human subjects. In order to verify this hypothesis, we determined, in a cohort of 622 subjects who took part in the SU.VI.MAX study $^{(21)}$, whether SFA intake modulated the expected association between blood lutein and zeaxanthin concentrations and fruit and vegetable intake. The answer was yes. Indeed, there was a positive significant association $(P=0.04)$ between fruit and vegetable intake and plasma lutein \pm zeaxanthin levels in subjects with low SFA intake, while there was no significant association $(P=0.87)$ in subjects with a high SFA intake. Nevertheless, the suggested effect of SFA intake on the relationship between fasting plasma concentrations of lutein and zeaxanthin and fruit and vegetable intake observed in the epidemiological association study was apparently not explained by the positive effect of SFA on lutein and zeaxanthin bioavailability suggested by the results of the present in vitro and rat studies. Indeed, the high intake of SFA was associated with lower plasma lutein and zeaxanthin concentrations in the second and third tertiles of fruit and vegetable intake. This apparent discrepancy may be due to potential limitations of the SU.VI.MAX study. Indeed, it is possible that there were some inaccuracies regarding dietary intake of the subjects that may have occurred as a result of the use of a self-administered FFQ. This apparent discrepancy can be also due to the fact that other mechanisms, as well as the positive effect of SFA on lutein and zeaxanthin bioavailability, are involved in the effect of SFA consumption on fasting blood lutein and zeaxanthin concentrations. For example, there might be an effect of blood SFA on uptake, or release, of xanthophylls by storage tissues, thus affecting blood concentrations of xanthophylls.
In conclusion, the results obtained from both in vitro and the rat studies suggest that SFA improve lutein and zeaxanthin bioavailability, mainly by increasing their bioaccessibility. Epidemiological associations, performed in a sample of the SU.VI.MAX study, support an interaction between SFA intake and fasting blood concentrations of lutein and zeaxanthin. However, higher intakes of SFA were not associated with higher fasting blood concentrations of these xanthophylls. This discrepancy may be, at least partly, ascribed to residual confounding in the human study. Thus, further experiments are necessary to conclude on the long-term effect of SFA consumption on lutein and zeaxanthin status.

\section{Supplementary material}

To view supplementary material for this article, please visit http://dx.doi.org/10.1017/S0007114512004813

\section{Acknowledgements}

The authors are grateful to Maria-Erica Lopez and Jean-Marc Feuerstein (C.F.R.E.M., Marseille, France), Jean-François Landrier, Aurélie Goncalves and Charlotte Sy (UMR INRA 1260, Marseille, France) for their technical help for the rat study. Thanks also to Marie-Josèphe Amiot-Carlin who put our team in touch with Bonduelle who funded the in vitro and rat experiments of the present study. The SU.VI.MAX study was funded by DGS (Ministry of Health) and supported by Médéric, Ipsen, MGEN, SODEXHO and Pierre Fabre. Work on the subset cohort of SU.VI.MAX was granted by ANR (no. ANR-05-PNRA010). The authors' contributions to the study were as follows: B. G., F. T., L. D., L. A., D. L. and P. B. designed the research; B. G., R. B. and M. N. conducted the research; E. K.-G., P. G. and S. H. constituted the SU.VI.MAX 2 investigators; B. G. and P. B. analysed data and performed statistical analysis; B. G. and P. B. wrote the paper and had primary responsibility for final content. All authors read and approved the final manuscript. No authors have any conflict of interest to declare.

\section{References}

1. Khachik F, Beecher GR, Goli M, et al. (1992) Separation and identification of carotenoids and their oxidation products in the extracts of human plasma. Anal Chem 64, 2111-2122.

2. Rao AV \& Rao LG (2007) Carotenoids and human health. Pharmacol Res 55, 207-216.

3. Maiani G, Caston MJ, Catasta G, et al. (2009) Carotenoids: actual knowledge on food sources, intakes, stability and bioavailability and their protective role in humans. Mol Nutr Food Res 53, Suppl. 2, S194-S218.

4. Bone RA, Landrum JT, Friedes LM, et al. (1997) Distribution of lutein and zeaxanthin stereoisomers in the human retina. Exp Eye Res 64, 211-218.

5. Junghans A, Sies H \& Stahl W (2001) Macular pigments lutein and zeaxanthin as blue light filters studied in liposomes. Arch Biochem Biophys 391, 160-164.

6. Krinsky NI (2002) Possible biologic mechanisms for a protective role of xanthophylls. J Nutr 132, 540S-542S. 
7. Snodderly DM (1995) Evidence for protection against agerelated macular degeneration by carotenoids and antioxidant vitamins. Am J Clin Nutr 62, 1448S-1461S.

8. Richer S, Stiles W, Statkute L, et al. (2004) Double-masked, placebo-controlled, randomized trial of lutein and antioxidant supplementation in the intervention of atrophic agerelated macular degeneration: the Veterans LAST study (Lutein Antioxidant Supplementation Trial). Optometry $\mathbf{7 5}$, 216-230.

9. Reboul E, Richelle M, Perrot E, et al. (2006) Bioaccessibility of carotenoids and vitamin $\mathrm{E}$ from their main dietary sources. J Agric Food Chem 54, 8749-8755.

10. van Het Hof KH, West CE, Weststrate JA, et al. (2000) Dietary factors that affect the bioavailability of carotenoids. $J$ Nutr 130, 503-506

11. Borel P (2003) Factors affecting intestinal absorption of highly lipophilic food microconstituents (fat-soluble vitamins, carotenoids and phytosterols). Clin Chem Lab Med 41, 979-994.

12. Yonekura L \& Nagao A (2007) Intestinal absorption of dietary carotenoids. Mol Nutr Food Res 51, 107-115.

13. Roodenburg AJ, Leenen R, van Het Hof KH, et al. (2000) Amount of fat in the diet affects bioavailability of lutein esters but not of alpha-carotene, beta-carotene, and vitamin $\mathrm{E}$ in humans. Am J Clin Nutr 71, 1187-1193.

14. Unlu NZ, Bohn T, Clinton SK, et al. (2005) Carotenoid absorption from salad and salsa by humans is enhanced by the addition of avocado or avocado oil. J Nutr 135, 431-436.

15. Lakshminarayana R, Raju M, Krishnakantha TP, et al. (2007) Lutein and zeaxanthin in leafy greens and their bioavailability: olive oil influences the absorption of dietary lutein and its accumulation in adult rats. J Agric Food Chem 55 , 6395-6400.

16. Huo T, Ferruzzi MG, Schwartz SJ, et al. (2007) Impact of fatty acyl composition and quantity of triglycerides on bioaccessibility of dietary carotenoids. J Agric Food Chem 55, 8950-8957.

17. Arvisenet G, Billy L, Poinot P, et al. (2008) Effect of apple particle state on the release of volatile compounds in a new artificial mouth device. J Agric Food Chem 56, 3245-3253.

18. Chantret I, Rodolosse A, Barbat A, et al. (1994) Differential expression of sucrase-isomaltase in clones isolated from early and late passages of the cell line Caco-2: evidence for glucose-dependent negative regulation. J Cell Sci 107, $213-225$

19. Salvini S, Charbonnier M, Defoort C, et al. (2002) Functional characterization of three clones of the human intestinal Caco-2 cell line for dietary lipid processing. Br J Nutr $\mathbf{8 7}$, 211-217

20. Reboul E, Abou L, Mikail C, et al. (2005) Lutein transport by Caco-2 TC-7 cells occurs partly by a facilitated process involving the scavenger receptor class B type I (SR-BI). Biochem J 387, 455-461.

21. Hercberg S, Galan P, Preziosi P, et al. (2004) The SU.VI.MAX Study: a randomized, placebo-controlled trial of the health effects of antioxidant vitamins and minerals. Arch Intern Med 164, 2335-2342.

22. Borel P, de Edelenyi FS, Vincent-Baudry S, et al. (2010) Genetic variants in BCMO1 and CD36 are associated with plasma lutein concentrations and macular pigment optical density in humans. Ann Med 43, 47-59.

23. Kesse-Guyot E, Castetbon K, Touvier M, et al. (2010) Relative validity and reproducibility of a food frequency questionnaire designed for French adults. Ann Nutr Metab 57 153-162.
24. Hercberg S (2005) Tables de composition des aliments SU.VI.MAX (Tables of Food Composition SU.VI.MAX) (in French). Paris: Economica.

25. Gleize B, Steib M, André M, et al. (2012) Simple and fast HPLC method for simultaneous determination of retinol, tocopherols, coenzyme Q10 and carotenoids in complex samples. Food Chem 134, 2560-2564.

26. Willet W, (1998) Nutritional Epidemiology, 2nd ed. New York: Oxford University Press.

27. Ito Y, Sasaki R, Suzuki S, et al. (1991) Relationship between serum xanthophyll levels and the consumption of cigarettes, alcohol or foods in healthy inhabitants of Japan. Int J Epidemiol 20, 615-620.

28. Rock CL, Thornquist MD, Neuhouser ML, et al. (2002) Diet and lifestyle correlates of lutein in the blood and diet. J Nutr 132, 525S-530S.

29. Gruber M, Chappell R, Millen A, et al. (2004) Correlates of serum lutein + zeaxanthin: findings from the Third National Health and Nutrition Examination Survey. J Nutr 134, 2387-2394

30. O'Connell O, Ryan L, O'Sullivan L, et al. (2008) Carotenoid micellarization varies greatly between individual and mixed vegetables with or without the addition of fat and fiber. Int J Vitam Nutr Res 78, 238-246.

31. Tyssandier V, Cardinault N, Caris-Veyrat C, et al. (2002) Vegetable-borne lutein, lycopene, and beta-carotene compete for incorporation into chylomicrons, with no adverse effect on the medium-term (3-wk) plasma status of carotenoids in humans. Am J Clin Nutr 75, 526-534

32. Reboul E, Thap S, Perrot E, et al. (2007) Effect of the main dietary antioxidants (carotenoids, gamma-tocopherol, polyphenols, and vitamin C) on alpha-tocopherol absorption. Eur J Clin Nutr 61, 1167-1173.

33. Carey MC \& Small DM (1970) The characteristics of mixed micellar solutions with particular reference to bile. Am J Med 49, 590-608.

34. Borel P, Armand M, Ythier P, et al. (1994) Hydrolysis of emulsions with different triglycerides and droplet sizes by gastric lipase in vitro - effect on pancreatic lipase activity. J Nutr Biochem 5, 124-133.

35. Reboul E, Thap S, Tourniaire F, et al. (2007) Differential effect of dietary antioxidant classes (carotenoids, polyphenols, vitamins C and E) on lutein absorption. Br J Nutr $\mathbf{9 7}$, 440-446.

36. Kanner J \& Lapidot T (2001) The stomach as a bioreactor: dietary lipid peroxidation in the gastric fluid and the effects of plant-derived antioxidants. Free Radic Biol Med 31, 1388-1395.

37. Lorrain B, Dangles O, Genot C, et al. (2010) Chemical modeling of heme-induced lipid oxidation in gastric conditions and inhibition by dietary polyphenols. J Agric Food Chem 58, 676-683.

38. Harris WS \& Muzio F (1993) Fish oil reduces postprandial triglyceride concentrations without accelerating lipid-emulsion removal rates. Am J Clin Nutr 58, 68-74.

39. van Greevenbroek MM, van Meer G, Erkelens DW, et al. (1996) Effects of saturated, mono-, and polyunsaturated fatty acids on the secretion of apo B containing lipoproteins by Caco- 2 cells. Atherosclerosis 121, 139-150.

40. Sauvant P, Mekki N, Charbonnier M, et al. (2003) Amounts and types of fatty acids in meals affect the pattern of retinoids secreted in human chylomicrons after a high-dose preformed vitamin A intake. Metabolism 52, 514-519.

41. Gleize B, Payet M, Esmail MH, et al. (2004) Docosahexaenoic acid-enriched egg phospholipids supplementation induces accretion of arachidonic acid in rat blood lipids. Cell Mol Biol 50, 861-867. 